\title{
Emotional Intelligence and Adaptive Success of Nurses Caring for People With Mental Retardation and Severe Behavior Problems
}

\author{
Linda Gerits, Jan J. L. Derksen, and Antoine B. Verbruggen
}

\begin{abstract}
The emotional intelligence profiles, gender differences, and adaptive success of 380 Dutch nurses caring for people with mental retardation and accompanying severe behavior problems are reported. Data were collected with the Bar-On Emotional Quotient Inventory, Utrecht-Coping List, UtrechtBurnout Scale, MMPI-2, and GAMA. Absence due to illness and job change were measured across a 2-year period. A clear relation between emotional intelligence and adaptive success was detected at an .01 level of significance. A negative correlation was found between emotional intelligence and both burnout and psychopathology. Emotional intelligence did not appear to be related to absence or job change. Based on these findings, training programs for nurses can possibly help to avoid employee burnout.
\end{abstract}

The concept of emotional intelligence was first introduced by Salovey and Mayer in 1990. They defined it as "the ability to monitor one's own and others' feelings and emotions, to discriminate among them, and to use this information to guide one's thinking and actions" (p. 189). After Goleman (1995) popularized the construct, it gained considerable public interest. Emotional intelligence is a relatively new term for a construct that complements the traditional concept of intelligence with an emphasis on the emotional, personal, and social contributions to intelligent behavior (Dawda \& Hart, 2000). Emotional intelligence is a mental aptitude, one that assists in intellectual processing, not a criterion that needs to be reached. People differ with respect to emotional intelligence (Mayer \& Salovey, 1993), which has three aspects: (a) the accurate appraisal and expression of emotion (in self and other people); (b) the adaptive regulation of emotions (in self and other people); and (c) the utilization of emotions to plan, create, and motivate action (Salovey, Hsee, \& Mayer, 1993). Much of the recent popular work suggests that emotional intelligence is highly predictive of an individual's general functioning and functioning within specific do- mains, such as career performance (Goleman, 1995).

The work of Bar-On (1997) in the domain of emotional intelligence culminated in the publication of the Emotional Quotient Inventory. He defined emotional intelligence as "an array of noncognitive capabilities, competencies, and skills that influence one's ability to succeed in coping with environmental demands and pressures" (p. 14). Emotional intelligence and emotional skills develop over time, change throughout the course of life, and can improve via training and remediation. In most of the research on emotional intelligence today, use is made of the Emotional Quotient Inventory, which divides emotional intelligence into five components and 15 subcomponents (Bar-On, 1997). Table 1 presents the scales and subscales as well as descriptions of each subscale.

In this paper we describe the application of the Bar-On Emotional Quotient Inventory to determine emotional intelligence of nurses who care for people with mental retardation and severe behavior problems. We were also interested in any gender differences in the nurses' emotional skills and in their adaptive success. These nurses were frequently confronted with serious self-injurious behaviors 
Table 1 Emotional Quotient Inventory

\begin{tabular}{|c|c|}
\hline Scale/Subscale & $\begin{array}{c}\text { Description of subscale: Ability } \\
\text { to ... }\end{array}$ \\
\hline \multicolumn{2}{|l|}{ Intrapersonal } \\
\hline Emotional Self-Awareness & Recognize and understand one's feelings \\
\hline Assertiveness & $\begin{array}{l}\text { Express feelings, beliefs, and thoughts and has ability to defend } \\
\text { one's rights in a nondestructive manner }\end{array}$ \\
\hline Self-Regard & Be aware of, understand, accept, and respect oneself \\
\hline Self-Actualization & Realize one's potential capacities \\
\hline Independence & $\begin{array}{l}\text { Be self-directed and self-controlled in one's thinking and actions } \\
\text { and free of emotional dependency }\end{array}$ \\
\hline \multicolumn{2}{|l|}{ Interpersonal } \\
\hline Empathy & Be aware of, understand, and appreciate feelings of others \\
\hline Interpersonal Relationship & $\begin{array}{l}\text { Establish and maintain mutually satisfying relationships charac- } \\
\text { terized by emotional closeness and the giving and receipt of } \\
\text { affection }\end{array}$ \\
\hline Social Responsibility & $\begin{array}{l}\text { Demonstrate oneself as a cooperative, contributing, and con- } \\
\text { structive member of one's social group }\end{array}$ \\
\hline \multicolumn{2}{|l|}{ Adaptation } \\
\hline Problem-Solving & $\begin{array}{l}\text { Identify and define problems as well as generate and implement } \\
\text { potentially effective solutions }\end{array}$ \\
\hline Reality Testing & $\begin{array}{l}\text { Assess the correspondence between what is subjectively experi- } \\
\text { enced and what objectively exists }\end{array}$ \\
\hline Flexibility & $\begin{array}{l}\text { Adjust one's emotions, thoughts, and behavior to changing situ- } \\
\text { ations and conditions }\end{array}$ \\
\hline \multicolumn{2}{|l|}{ Stress Management } \\
\hline Stress Tolerance & $\begin{array}{l}\text { Withstand adverse events and stressful situations without "fall- } \\
\text { ing apart" by actively and positively coping with stress }\end{array}$ \\
\hline Impulse Control & Resist or delay an impulse, drive, or temptation to act \\
\hline \multicolumn{2}{|l|}{ General Mood } \\
\hline Happiness & $\begin{array}{l}\text { Feel satisfied with one's life, enjoy oneself and others, and have } \\
\text { fun }\end{array}$ \\
\hline Optimism & $\begin{array}{l}\text { Look at the brighter side of life and maintain a positive atti- } \\
\text { tude, even in the face of adversity }\end{array}$ \\
\hline
\end{tabular}

aSubscale descriptions are based on Bar-0n (1997, pp. 44-47).

(SIBs), aggression, and/or destructive behaviors as determined by scores of 3 or 4 on a Dutch consensus protocol regarding problem behavior. The behaviors are characterized by a very severe or extreme intensity, causing serious damage to the client, his or her caregiver, or other service users and a highly frequent, almost daily, occurrence as well as by radical interventions required to prevent or stop them (e.g., high doses of medication, frequent isolation, or restraint). The behavior problems have a long-lasting character, and there is a lack of effective treatment procedures in many cases ( $\mathrm{Kra}$ mer, 1995).

Given the challenging nature of the clients they are taking care of, the demands on the care staff are quite high and clearly constitute an antecedent to stress. Various negative emotional reactions can occur, accompanied by increased levels of 
perceived stress. Extra time and energy are needed to analyze, solve, and prevent dangerous situations, which can also lead to a higher workload. Physical intervention to ensure the safety of the service user or others, for example, is a daily occurrence in approximately one third of cases (Bromley \& Emerson, 1995).

The extensive efforts of the personnel to treat the behavior problems of such clients are not always successful. About $25 \%$ of all behavior problems are treated quite effectively, about $50 \%$ fairly effectively, and about $25 \%$ only questionably. Even with successful intervention, moreover, it is unlikely that the behavior problems totally disappear (Didden, Duker, \& Korzilius, 1997). Along these lines, personnel have indicated that the most common sources of stress are the enduring character of the client's behavior, the absence of any effective method for dealing with the behavior, the unpredictability of the behavior, and difficulties understanding the person's behavior.

Personnel also typically experience a range of emotions in response to an episode of challenging behavior, for example, annoyance, anger, or fear after an episode of aggression or sadness, despair, or disgust after an episode involving self-injury. Further, according to a number of researchers, such emotions or reactions suggest a process of burnout (Bromley \& Emerson, 1995).

The occupational culture of nurses dictates control of their affective reactions to tragic or violent circumstances (Bar-On, Brown, Kirkcaldy, \& Thomé, 2000). The nurses in our study were certainly confronted by such circumstances, which can create emotional conflict. In addition, a gendered dimension of this occupation appears to produce very different demands on nursing personnel. On the one hand, there is the perception that feminine qualities are best suited for the emotional aspects of nursing. On the other hand, clearly masculine characteristics are needed for the care of clients with severe behavior problems (i.e., to help control their aggressive tendencies). In terms of emotional intelligence, which encompasses a broad array of factors and skills that contribute to successful functioning, it is interesting to examine the possibly gender-specific skills that appear to be essential for the particular nursing profession being considered here and the way these skills may be related with adaptive success or symptoms of nonadaptability.

With regard to a person's adaptive success, coping and general intelligence are known to play a crucial role. Coping refers to the efforts to manage or control a situation that a person considers stressful, overtaxing, or challenging (Lazarus \& Folkman, 1984). Emotional intelligence influences the selection and control of coping strategies under challenging and/or stressful circumstances (Matthews \& Zeidner, 2000). Conversely, effective coping is often seen as a major component of emotional intelligence. In a similar vein, the flexible application of knowledge plays a crucial role in adaptive success (Sternberg, 1985). That is, a key attribute of general intelligence is its transferability: The intelligent person adapts existing knowledge to deal with a novel problem.

In discussions of emotional intelligence, it is sometimes unclear whether the focus is on the intelligent use of emotions or on emotions as a form of intelligence. Theoretically, these are different concepts, although in practice they may be difficult to disentangle (Averill, 2000). Furthermore, within the context of the present study, burnout, psychopathology, absenteeism due to illness, and job change are considered symptoms of nonadaptability. Burnout can be conceptualized as the result of failed adaptation to chronically demanding and stressful situations. When helplessness, feelings of impotence, and defensive coping styles emerge due to inability to influence such situations on several occasions, chronic stress can turn into burnout (Maslach \& Jackson, 1986). Burnout also appears to occur predominantly in professions involving close contact with other people, such as the health care and teaching professions (Leiter \& Maslach, 1988). Psychopathology is also considered a symptom of nonadaptability. Emotional intelligence is known to be positively related with measures of emotional stability and negatively with measures of neuroticism and psychopathology (Dawda \& Hart, 2000).

On the basis of above information, we expected that nurses scoring high on the Emotional Quotient Inventory would use more effective coping styles than would those scoring low. We measured coping styles with the Utrecht-Coping List (Schreurs, Willige, Brosschot, Tellegen, \& Graus, 1993). We predicted further that they would have higher scores on general intelligence, as measured by the General Ability Measure for Adults_GAMA (Naglieri \& Bardos, 1997), lower scores on burnout (as measured by the Utrecht-Burnout Scale (Schaufeli \& Dierendonck, 2000), less symptoms of psychopathology in terms of the Minnesota Multiphasic Personality Inventory-2-MMPI-2 (Butcher, Dahls- 
trom, Graham, Tellegen, \& Kaemmer, 1989), and lower rates of absence due to illness or job change. Similarly, we expected positive correlations between the composite Adaptation scale from the Emotional Quotient Inventory and coping. A negative correlation was expected to characterize the relation between scores on the Stress Management composite scale of the Emotional Quotient Inventory and burnout. In addition, we expected negative correlations between General Mood composite scale and psychopathology.

The present study was conducted within the context of a larger research program aimed to develop a profile for the selection and training of nurses to care for people with mental retardation and accompanying severe behavior problems.

\section{Method}

\section{Participants}

Data were collected from 380 nurses (40\% male) working in 56 Dutch residential facilities for people with mental retardation. The subjects were selected from a sample of 436 respondents (response rate of $83 \%$ ) on the basis of valid emotional intelligence scores ( 55 profiles were invalid). Their average age was 33.3 years (range $=20$ to 59). All participants were working with individuals who had severe behavior problems (i.e., self-injurious, aggressive, or destructive behaviors) as determined by scores of 3 or 4 on a Dutch consensus protocol regarding problem behavior (Kramer, 1995). The educational level of the participating nurses was as follows: $55.4 \%$ had technical and vocational training, $41.7 \%$ had a college education; and $1.8 \%$ were university graduates. The remaining $1.1 \%$ had no secondary education. All of the participants worked at least 18 hours a week $(50 \%$ contract for direct client care), had been employed for at least half a year, and had a regular as opposed to temporary contract. In other words, we included only nurses who spent at least half of their working week in the professional environment, were already accustomed to the work, and were not working on an initial probationary basis or under a temporary contract.

The majority of the participants (83.7\%) were working in a residential group home setting. The remaining participants worked in either a residential day-care setting $(13.3 \%)$ or both types of settings $(2.8 \%)$. The average contract was for 32.2 hours a week (standard deviation $[S D]=4.8$ ). The participants reported working an average of 10.3 years with people who had mental retardation and for an average of 6.1 years with people who had mental retardation accompanied by severe behavior problems.

\section{Instruments}

The Dutch version of the Bar-On Emotional Quotient Inventory (1997), as developed by Derksen, Jeuken, and Klein Herenbrink (1998), was used to measure emotional intelligence. This inventory consists of a 15-factor scale spanning the domains of Intrapersonal Abilities, Interpersonal Skills, Adaptability, Stress-Management Capacity, and General Mood. In addition, two validity scales are included to identify overly positive or overly negative self-representations, and a response-consistency score was used to indicate the validity of the results. The Emotional Quotient Inventory contains 133 numbered statements, with response categories ranging from 1 (never) to 5 (daily). An example of an item is "I feel sure of myself in most situations." Completion of the Emotional Quotient Inventory takes 20 to 30 minutes. Similar to IQs, Emotional Quotient Inventory raw scores are converted to standard scores, with 100 as the mean and the unit of SD set at 15 points. Considerable support has been found for the reliability and validity of the measure. The average Cronbach alpha coefficients for the different subscales have been found to range from .69 to .86 . The average test-retest reliability coefficients after 1 and 4 months have been found to be .85 and .75 , respectively. The enormous number of empirical findings presented in the manual convincingly show the Bar-On Emotional Quotient Inventory to be a reliable and valid instrument. For example, the construct validity has been examined in 16 countries, and the Emotional Quotient Inventory has been found to tap a broad range of related emotional constructs. The structural properties of the measure are also good. The Inventory taps a fairly broad range of related emotional constructs, and no gender bias has been detected (Dawda \& Hart, 2000).

The Utrecht-Coping List was used to determine coping style. This instrument contains 47 items divided into seven scales: Active Dealing, Palliative Reaction, Avoidance, Social Support Seeking, Passive Reaction, Expression of Emotions, and Comforting Thoughts. Possible answers are rarely, sometimes, frequently, and very often. Scores are transformed into $T$ scores along a 5-point scale ranging from 1 (very low, $\leq 5$ th percentile) to 5 
(very high, $\geq 95$ th percentile). Considerable support has been found for the reliability of the scales, which have also proved quite stable over time, Pearson $r=.54$. The average Cronbach alpha coefficients for the different subscales have been found to range from .61 to .78 . The construct, factorial, and predictive validity are sufficient. The UtrechtCoping List scale scores can be seen as mediating between "trait" measures and "state" measures, which is in accordance with the theoretical formulation of coping as a personality style (Schreurs et al., 1993).

The Dutch version of the Naglieri and Bardos (1997) GAMA, as developed by Derksen, Egger, and Bögels (1998), is a brief, self-administered, and nonverbal measure of general intelligence using nonverbal means, which makes the measure independent of both verbal content and instructional factors. The instrument consists of 66 items organized into four types or subscales: Matching, Sequences, Analogies, and Construction. Each item has a response set of five possibilities. The test results include a total IQ, with a mean of 100 and an SD of 15. The GAMA must be completed within 25 minutes. The test has been standardized for the Dutch population using a census-based sample of 874 adults stratified by age, gender, ethnic background, educational level, and geographic region. Research regarding the validity of the GAMA has yielded favorable results: Correlation coefficients between GAMA and the Wechsler Adult Intelligence Scale, Revised (WAIS-R) and the KaufmanBrief Intelligence Test (K-BIT), for example, were highly associated, $r s>$.70. Internal reliability coefficients for the four subscales range from .65 to .81 , and test-retest reliability estimates for the GAMA IQs range from .79 to .94 (Lassiter \& Matthews, 1999; Naglieri \& Bardos, 1997).

The 20-item version of the Utrecht-Burnout Scale was used to measure burnout (Schaufeli \& Dierendonck, 2000). This scale is a Dutch version of the Maslach Burnout Inventory (Maslach \& Jackson, 1986) and contains three subscales pertaining to Emotional Exhaustion, Depersonalization, and Personal Accomplishment, with 8, 5, and 7 items, respectively. The response rating categories range from 0 (never) to 6 (always/daily). Completion of the Utrecht-Burnout Scale takes 10 to $15 \mathrm{~min}$ utes. The scales are internally consistent: the Emotional Exhaustion and Personal Accomplishment both exceed the .70 criterion, the internal consistency of the Depersonalization scale is a little bit lower (.66). The Utrecht-Burnout Scale has proved relatively stable over time: The test-retest reliability is .69 for Emotional Exhaustion, .62 for Depersonalization, and .71 for Personal Accomplishment across a 2-year period of time. The factor validity has also proved good (Schaufeli \& Dierendonck, 2000).

The Dutch version of the Butcher et al. (1989) Minnesota Multiphasic Personality Inventory-2MMPI-2 (Derksen, Mey, Sloore, \& Hellenbosch, 1995) was used to measure personality and psychopathology. The Inventory contains 567 numbered statements with the response categories agree and do not agree. It takes 1 to 1.5 hours to complete. The resulting personality profile is based on $8 \mathrm{va}$ lidity scales, 10 clinical scales, and 15 content scales. The clinical scales are (1) Hypochondriasis, (2) Depression, (3) Hysteria, (4) Psychopathic Deviate, (5) Masculinity-Femininity, (6) Paranoia, (7) Psychasthenia, (8) Schizophrenia, (9) Hypomania, and (10) Social Introversion. The content scales are developed to measure Anxiety, Fears, Obsessiveness, Depression, Health Concerns, Bizarre Mention, Anger, Cynicism, Antisocial Practices, Type A Behavior, Low Self-Esteem, Social Discomfort, Family Problems, Work Interference, and Negative Treatment Indicators. (For a description of the scales, see Graham, 2000.) After raw scores have been corrected, standardized scores can be computed for the clinical scales and the content scales. Apart from the discussion of these scales, interpretations are provided for 2-and 3-point code types based on the highest clinical scales in a profile. Various subscales and supplementary scales are used to measure such aspects as ego strength, marital distress, hostility, alcoholism, and posttraumatic stress disorder.

The MMPI-2 is well-standardized in terms of materials, administration, and scoring. The clinical scales of the MMPI were constructed according to empirical keying procedures, when patients responded differently to items than did individuals who were not being treated clinically. Psychometric qualities of the MMPI-2 are sufficient. Test-retest reliability coefficients range from .43 (Paranoia) to .88 (Schizophrenia) for the clinical scales, from .30 (True Response Inconsistency) to .90 (Posttraumatic Stress Disorder) for the supplementary scales, and from .67 (Antisocial Practices) to .91 (Depression) for the content scales. Internal reliability coefficients for the clinical scales range from .32 (Masculinity-Femininity) to .85 (Psychasthenia), from 
.15 (Overcontrolled Hostility) to .89 (Posttraumatic Stress Disorder) for the supplementary scales and from .64 (Fears, Antisocial Practices) to .84 (Depression) for the content scales (Graham, 2000). Normative samples for many countries are available. The Dutch norms are based on a representative sample of 1,244 individuals (Derksen et al., 1995).

Absence due to illness was measured separately for each of the 2 calendar years immediately following completion of the Emotional Quotient Inventory. In order to do this, we were provided with data by the personnel departments from the relevant residential facilities. The measures of absence derived were number of episodes of illness and absence rate (total number of days absent through illness controlled for the amount of hours worked per week). Absences due to participation in study or pregnancy were not included in this measure.

Job change was measured across a 2-year period. The data were provided by a spokesperson or personnel manager from each of the relevant residential facilities. Those nurses who had changed jobs or resigned were approached to complete a 15item questionnaire concerning their reasons for resignation and new career choice.

\section{Procedure}

The participants in this study were asked to take part in a larger 2-year longitudinal study of the personal factors relating to burnout in nurses working with people who have mental retardation and accompanying severe behavior problems. We first wrote to the boards of 96 residential facilities for people with mental retardation in the Netherlands. After 56 residential facilities (response rate of $67.1 \%$ ) agreed to participate, those nurses who met the selection criteria were approached by their managers. This recruitment process was coordinated by a spokesperson who arranged for the nurses from each residential facility to gather on a specific date to complete all of the questionnaires (response rate of $83 \%$ ). The data collection was further supervised by the first or third author. The data for the current study were from the first point of data collection (Time 1) in the fall and winter of 1999.

\section{Analyses}

First, the Emotional Quotient Inventory protocols were validated by filtering for consistency (Inconsistency Index $>12$ ), positive impression (Positive Impression Score $>130$ ), and negative impression (Negative Impression Score $>130$ ). The final sample consisted of 380 valid Emotional Quotient Inventory protocols (87.2\%); 56 of the original 436 were judged to be invalid. For the valid protocols, bivariate correlations were computed between the standardized Emotional Quotient Inventory total and Emotional Quotient Inventory composite scores and the following standardized variables: Utrecht-Coping List subscales (7), GAMA total score (1), Utrecht-Burnout Scale dimensions (3), MMPI-2 Clinical (10), and MMPI-2 Content (15) Scales. In addition, bivariate correlations were computed between the Emotional Quotient Inventory Total and Emotional Quotient Inventory Composite scores and the number of illness episodes, total number of days absent due to illness, and the illness index rates. Binary logistic regression was computed between Emotional Quotient Inventory Total, Emotional Quotient Inventory Composite scores, and job change $(1 / 0)$. Given the number of correlations performed, a significance level of .01 was used.

\section{Results}

\section{Gender Differences}

As shown in Table 2, there were several significant gender differences in the emotional intelligence scores of these nurses. Male nurses scored significantly higher than did female nurses on the Intrapersonal composite scale, Assertiveness subscale, and Self-Regard subscale. They also scored significantly higher on the Stress Management composite scale and the Stress Tolerance subscale. Female nurses scored significantly higher on the Interpersonal composite scale and all of the associated subscales of Empathy, Interpersonal Relationship, and Social Responsibility. No significant difference was found between the men and women for the Emotional Quotient Inventory total.

\section{Emotional Intelligence and Coping}

To determine whether nurses with a higher level of emotional intelligence used different coping styles than did nurses with a lower level, we computed correlations between their Emotional Quotient Inventory total scores and their Utrecht-Coping List scores. The results are presented in Table 3. As can be seen, the male nurses with a higher Emotional Quotient Inventory total score also had a significantly higher score for the Active Dealing coping style and a significantly lower score for the 
Table 2 Distribution of Emotional Quotient Inventory Scales by Gender

\begin{tabular}{|c|c|c|c|c|c|}
\hline \multirow[b]{2}{*}{ Scale/Subscale } & \multicolumn{2}{|c|}{ Men $(n=146)$} & \multicolumn{2}{|c|}{ Women $(n=234)$} & \multirow[b]{2}{*}{ Contrast ${ }^{\mathrm{b}}$} \\
\hline & Mean & $S D$ & Mean & $S D$ & \\
\hline Total & 107.24 & 10.66 & 107.15 & 11.54 & 0.08 \\
\hline Intra & 107.13 & 10.51 & 104.61 & 12.94 & $1.98^{*}$ \\
\hline ES & 105.84 & 12.75 & 107.75 & 13.99 & -1.34 \\
\hline AS & 109.01 & 12.12 & 104.88 & 15.50 & $2.73^{*}$ \\
\hline SR & 100.68 & 10.44 & 96.30 & 12.39 & $3.55^{*}$ \\
\hline SA & 107.34 & 11.16 & 107.98 & 11.60 & -0.53 \\
\hline IN & 106.31 & 11.50 & 102.90 & 13.43 & $2.54^{*}$ \\
\hline Inter & 100.62 & 12.55 & 106.70 & 10.50 & $-5.09 *$ \\
\hline EM & 99.02 & 11.81 & 104.39 & 10.63 & $-4.59 *$ \\
\hline IR & 104.90 & 12.88 & 109.28 & 11.06 & $-3.52^{*}$ \\
\hline RE & 95.99 & 13.93 & 101.99 & 11.80 & $-4.49^{*}$ \\
\hline Adapt & 105.55 & 11.58 & 105.67 & 12.58 & -0.09 \\
\hline PS & 99.62 & 13.74 & 98.89 & 13.59 & 0.51 \\
\hline RT & 107.89 & 12.72 & 108.21 & 12.78 & -0.23 \\
\hline $\mathrm{FL}$ & 104.44 & 13.90 & 105.16 & 13.64 & -0.50 \\
\hline STRES & 109.08 & 11.73 & 106.26 & 12.76 & $2.16^{*}$ \\
\hline ST & 110.49 & 10.66 & 106.15 & 10.93 & $3.79 *$ \\
\hline IC & 103.88 & 12.63 & 103.59 & 14.70 & 0.20 \\
\hline MOOD & 108.01 & 10.20 & 107.90 & 11.07 & 0.09 \\
\hline $\mathrm{HA}$ & 107.65 & 10.76 & 108.59 & 10.88 & -0.83 \\
\hline $\mathrm{OP}$ & 106.45 & 10.92 & 105.04 & 11.23 & 1.21 \\
\hline
\end{tabular}

antra, Intrapersonal; ES, Emotional Self-Awareness; AS, Assertiveness; SR, Self-Regard; SA, Self-Actualization; IN, Independence; INTER, Interpersonal; EM, Empathy; IR, Interpersonal Relationship; RE, Social Responsibility; ADAPT, Adaptation: PS, Problem Solving; RT, Reality Testing; FL, Flexibility; STRES, Stress Management; ST, Stress Tolerance; IC, Impulse Control; MOOD, General Mood; HA, Happiness; OP, Optimism. ${ }^{\mathrm{b} A l l} d f \mathrm{f}=378$. ${ }^{*} p<.05$.

Avoidance and Passive Reaction coping styles. For the female nurses, a similar pattern with even stronger correlations was found. In addition, a significant positive correlation was found for the female nurses between their Emotional Quotient Inventory total scores and use of a Social Support Seeking coping style. Nurses with higher emotional intelligence, thus, tend to generally use a more active coping style to deal with problems and tend to react less passively and with less avoidance than do nurses with lower emotional intelligence. In addition, female nurses with higher emotional intelligence tend to seek social support under such circumstances more frequently than do those with lower emotional intelligence.

As can be seen from inspection of Table 3 as well, the Emotional Quotient Inventory composite scale scores of the nurses also correlated signifi- cantly with their scores for several of the coping variables, $p$ s $<.01$. With the exception of the Intrapersonal and Adaptation Emotional Quotient Inventory composite scales for men, higher scores on all of the Emotional Quotient Inventory composite scales correlated for both the male and female nurses, with higher scores on the use of an Active Dealing coping style. In addition, higher scores on the Stress Management composite scale for female nurses were associated with higher scores for use of a Palliative Reaction coping style. Lower scores on the Intrapersonal and Adaptation composite scales for both male and female nurses were associated with higher scores for the use of an Avoidance coping style. Furthermore, higher scores on the Intrapersonal, Interpersonal, Adaptation, and General Mood composite scales for women were associated with higher use of a Social Support Seeking coping 
Table 3 Emotional Quotient Inventory Scales: Correlations With Coping Subscales by Gender

\begin{tabular}{llllllll}
\hline & \multicolumn{7}{c}{ Utrecht Coping List $^{\mathrm{b}}$} \\
\cline { 2 - 7 } Gender/Scale & ACT & PAL & AV0 & SOC & PAS & EXP & COM \\
\hline Men $(n=146)$ & & & & & & & \\
TOTAL & $.34^{* *}$ & -.04 & $-.23^{* *}$ & .09 & $-.31^{* *}$ & -.02 & -.06 \\
INTRA & .21 & .04 & $-.29^{* *}$ & .16 & $-.25^{* *}$ & .15 & -.03 \\
INTER & $.31^{* *}$ & .05 & -.04 & $.22^{* *}$ & -.11 & -.03 & .10 \\
ADAPT & $.33^{* *}$ & -.09 & $-.23^{* *}$ & -.02 & $-.28^{* *}$ & -.04 & -.15 \\
STRES & .20 & $-.23^{* *}$ & -.09 & $-.22^{* *}$ & $-.29^{* *}$ & $-.30^{* *}$ & -.19 \\
MOOD & $.21^{* *}$ & .01 & -.13 & .15 & $-.31^{* *}$ & .01 & -.02 \\
Woman $(n=233)$ & & & & & & \\
TOTAL & $.43^{* *}$ & -.02 & $-.23^{* *}$ & $.33^{* *}$ & $-.45^{* *}$ & -.01 & -.08 \\
INTRA & $.38^{* *}$ & -.01 & $-.31^{* *}$ & $.34^{* *}$ & $-.39^{* *}$ & .13 & -.09 \\
INTER & $.20^{* *}$ & .06 & -.13 & $.36^{* *}$ & -.15 & -.05 & -.01 \\
ADAPT & $.36^{* *}$ & -.09 & $-.24^{* *}$ & $.23^{* *}$ & $-.43^{* *}$ & -.07 & -.06 \\
STRES & $.31^{* *}$ & -.11 & .02 & .00 & $-.35^{* *}$ & $-.23^{* *}$ & -.12 \\
MOOD & $.38^{* *}$ & .10 & -.08 & $.30^{* *}$ & $-.38^{* *}$ & .05 & .04 \\
\hline
\end{tabular}

aINTRA, Intrapersonal; INTER, Interpersonal; ADAPT, Adaptation; STRES, Stress Management; MO0D, General Mood. 'DACT, Active Dealing; PAL, Palliative Reaction; AVO, Avoidance; SOC, Social Support Seeking; PAS, Passive Reaction; EXP, Expression of Emotions; COM, Comforting.

${ }^{* *} p<.01$ (two-tailed).

style. For men, only their scores on the Interpersonal and Stress Management composite scales significantly correlated with higher scores for the use of Active Dealing and Social Support Seeking coping styles.

On average, the Emotional Quotient Inventory composite scales were most strongly related to a Passive Reaction and an Active Dealing coping style. Finally, higher scores on the Stress Management composite scale only correlated significantly with higher scores for an expression of Emotion coping style, and no Emotional Quotient Inventory composite scale correlated significantly with having Comforting Thoughts as a coping style.

\section{Emotional Intelligence and General} Intelligence

To test whether nurses with a higher level of emotional intelligence also show a higher level of general intelligence, we computed the correlations for the Emotional Quotient Inventory total and composite scale scores with the GAMA total scores for the nurses. In Table 4, the correlations for male and female nurses are shown. Only women with a higher score on the Emotional Quotient Inventory total scale and composite scales of Intrapersonal,
Interpersonal, and General Mood showed significant positive correlations with their GAMA total scores. Although these correlations were significant, they were not high (ranging from .17 to .22), which suggests that only a very minimal relation exists between general intelligence and emotional intelligence for female nurses.

Table 4 Emotional Quotient Inventory Scales: Correlations With General Intelligence (GAMA)

\begin{tabular}{lcc}
\hline & \multicolumn{2}{c}{ GAMA } \\
\cline { 2 - 3 } Scale $^{\mathrm{a}}$ & Men $^{\mathrm{b}}$ & Women $^{\mathrm{c}}$ \\
\hline TOTAL & .16 & $.20^{* *}$ \\
INTRA & .14 & $.17^{* *}$ \\
INTER & .13 & $.18^{* *}$ \\
ADAPT & .13 & .11 \\
STRES & .04 & .08 \\
MOOD & .18 & $.22^{* *}$ \\
\hline
\end{tabular}

Note. GAMA $=$ General Ability Measure for Adults. aINTRA, Intrapersonal; INTER, Interpersonal; ADAPT, Adaptation; STRES, Stress Management; M00D, General Mood. ${ }^{b} N=146 .{ }^{c} N=234$.

${ }^{* *} p<.01$ (two-tailed). 


\section{Emotional Intelligence and Burnout}

To test whether nurses with lower emotional intelligence have greater burnout, we computed the correlations of the Emotional Quotient Inventory total and composite scale scores with the UtrechtBurnout Scale scores. The results are presented in Table 5. For the male nurses, the only significant correlation between emotional intelligence and burnout was found for Personal Accomplishment. Male nurses with higher scores on the Emotional Quotient Inventory total, Interpersonal composite scale, and General Mood composite scale also scored significantly higher on the Personal Accomplishment aspect of burnout. In other words, male nurses with a higher level of emotional intelligence, better interpersonal skills, and a better general mood reported greater feelings of personal competence (and, thus, less burnout).

The female nurses in this sample showed very different results. Lower levels of emotional intelligence in general and lower scores on the Intrapersonal, Adaptation, Stress Management, and General Mood composite scales were associated with

Table 5 Emotional Quotient Inventory Scales: Correlations With the Dimensions of Burnout (Utrecht-Burnout Scales) by Gender

\begin{tabular}{lcll}
\hline & \multicolumn{3}{c}{ Utrecht-Burnout Scale } \\
\cline { 2 - 4 } Gender/Scale & EE & DEP & COM \\
\hline Men $(n=146)$ & & & \\
TOTAL & -.18 & -.12 & $.25^{* *}$ \\
INTRA & -.13 & -.03 & .18 \\
INTER & -.04 & -.16 & $.22^{* *}$ \\
ADAPT & -.18 & -.12 & .20 \\
STRES & -.20 & -.09 & .16 \\
MOOD & -.21 & -.04 & $.23^{* *}$ \\
Women $(n=234)$ & & \\
TOTAL & $-.33^{* *}$ & $-.32^{* *}$ & $.38^{* *}$ \\
INTRA & $-.28^{* *}$ & $-.21^{* *}$ & $.29^{* *}$ \\
INTER & -.13 & $-.19^{* *}$ & $.23^{* *}$ \\
ADAPT & $-.36^{* *}$ & $-.31^{* *}$ & $.35^{* *}$ \\
STRES & $-.22^{* *}$ & $-.31^{* *}$ & $.27^{* *}$ \\
MOOD & $-.18^{* *}$ & $-.22^{* *}$ & $.28^{* *}$ \\
\hline
\end{tabular}

aINTRA, Intrapersonal; INTER, Interpersonal; ADAPT, Adaptation; STRES, Stress Management; MOOD, General Mood. ${ }^{\mathrm{b} E E}$, Emotional Exhaustion; DEP, Depersonalization; and COM, Personal Accomplishment. ${ }^{* *} p<.01$ (two-tailed). higher scores on the Emotional Exhaustion dimension of burnout. Lower scores on the Emotional Quotient Inventory total and all five composite scales correlated significantly with higher scores on the Depersonalization dimension of burnout. Conversely, higher scores on the Emotional Quotient Inventory total and all five of the composite scales correlated significantly with lower scores on the Personal Accomplishment dimension of burnout.

In sum, emotional intelligence generally correlated significantly with the Personal Accomplishment dimension of burnout for the male nurses and all three dimensions of burnout for the female nurses.

\section{Emotional Intelligence and Psychopathology}

To test whether nurses with a higher level of emotional intelligence show less psychopathology and, thus, lower scores on the MMPI-2, we computed the correlations between their Emotional Quotient Inventory total and composite scale scores, on the one hand, and their scores for the MMPI-2 Clinical and Content scales, on the other hand. The results for the MMPI-2 Clinical scales will first be discussed and are presented in Table 6 . Given the large number of correlations calculated, only the highest $(\leq .30)$ are discussed here. For the male nurses, significant negative correlations were found for the Emotional Quotient Inventory total, Intrapersonal composite scale, and General Mood composite scale with the MMPI-2 Clinical Scale 0 (Social Introversion); that is, male nurses scoring lower on this MMPI-2 scale who tend to be more extraverted, tend to have a higher level of emotional intelligence, a better ability to express their feelings, better social skills, and a better general mood. A similar pattern of even higher correlations was shown for the female nurses scoring lower on this MMPI-2 scale. For the female nurses as well, lower scores for emotional intelligence in general, the Intrapersonal composite scale, and the General Mood composite scale were also associated with higher scores on the MMPI-2 Clinical scales of 2 and 7-indicating Depression and Psychastheniathat characterize people with negative feelings about themselves and people who tend to worry, feel depressed, and feel anxious. These same individuals tend to anticipate problems before they occur and to overreact to minor stress.

The results for the MMPI-2 Content scales are presented in Table 7. As can be seen, emotional intelligence relates significantly to many of the 


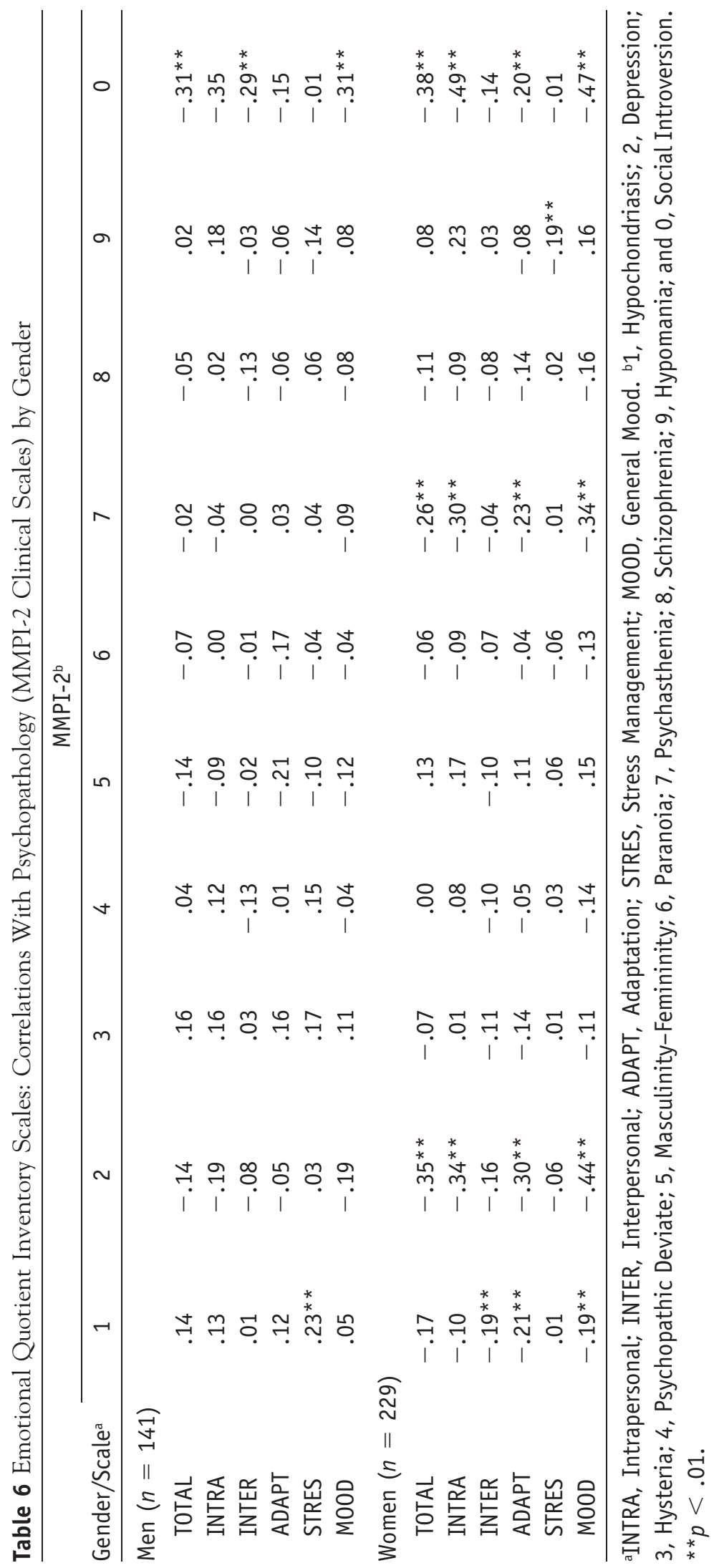


Table 7 Emotional Quotient Inventory Scales: Correlations With Psychopathology (MMPI-2 Content Scales) by Gender

\begin{tabular}{|c|c|c|c|c|c|c|}
\hline \multirow{2}{*}{$\begin{array}{l}\text { Gender/ } \\
\text { Scale }^{a}\end{array}$} & \multicolumn{6}{|c|}{ Emotional Quotient Inventory } \\
\hline & TOTAL & INTRA & INTER & ADAPT & STRES & MOOD \\
\hline \multicolumn{7}{|c|}{$\operatorname{Men}(n=141)$} \\
\hline ANX & $-.25^{* *}$ & -.21 & -.03 & -.22 & $-.28 * *$ & $-.23^{* *}$ \\
\hline FRS & -.17 & -.21 & .01 & -.12 & -.21 & -.07 \\
\hline OBS & -.19 & -.20 & .10 & -.21 & -.29 & -.12 \\
\hline DEP & $-.36^{* *}$ & $-.27^{* *}$ & -.21 & $-.27 * *$ & $-.25^{* *}$ & $-.39 * *$ \\
\hline HEA & -.07 & .03 & -.04 & -.11 & -.11 & -.05 \\
\hline $\mathrm{BIZ}$ & $-.28 * *$ & -.17 & -.18 & $-.29 * *$ & $-.30 * *$ & -.16 \\
\hline ANG & $-.28 * *$ & -.09 & -.14 & $-.30 * *$ & $-.46^{* *}$ & -.20 \\
\hline CYN & -.26 ** & -.13 & -.17 & $-.25 * *$ & $-.29 * *$ & -.17 \\
\hline ASP & $-.25^{* *}$ & -.09 & $-.25^{* *}$ & $-.25^{* *}$ & $-.27^{* *}$ & -.15 \\
\hline TPA & $-.32 * *$ & -.18 & -.20 & $-.29 * *$ & $-.43 * *$ & -.15 \\
\hline LSE & $-.33 * *$ & $-.38 * *$ & -.13 & $-.22^{* *}$ & -.15 & $-.29 * *$ \\
\hline SOD & $-.29 * *$ & $-.29 * *$ & $-.34^{* *}$ & -.17 & .01 & $-.28 * *$ \\
\hline FAM & $-.24^{* *}$ & -.17 & -.21 & $-.23 * *$ & -.12 & -.20 \\
\hline WRK & $-.43 * *$ & $-.35^{* *}$ & -.19 & $-.42^{* *}$ & $-.34^{* *}$ & $-.36^{* *}$ \\
\hline TRT & $-.38 * *$ & $-.28 * *$ & $-.32 * *$ & $-.25^{* *}$ & $-.28 * *$ & $-.33^{* *}$ \\
\hline \multicolumn{7}{|c|}{ Women $(n=229)$} \\
\hline ANX & $-.44^{* *}$ & $-.39 * *$ & -.10 & -.40 ** & $-.38^{* *}$ & $-.37^{* *}$ \\
\hline FRS & -.11 & -.10 & -.02 & -.14 & -.10 & -.06 \\
\hline OBS & $-.48 * *$ & $-.45^{* *}$ & -.13 & $-.43^{* *}$ & $-.37 * *$ & $-.34 * *$ \\
\hline DEP & $-.51^{* *}$ & $-.47^{* *}$ & $-.19 * *$ & $-.43^{* *}$ & $-.29 * *$ & $-.52^{* *}$ \\
\hline HEA & $-.27^{* *}$ & -.15 & $-.21^{* *}$ & -.30 ** & $-.19 * *$ & $-.18^{* *}$ \\
\hline $\mathrm{BIZ}$ & $-.21 * *$ & -.11 & -.06 & $-.26^{* *}$ & $-.29 * *$ & -.09 \\
\hline ANG & $-.30 * *$ & -.10 & -.17 & $-.31^{* *}$ & $-.50 * *$ & $-.18^{* *}$ \\
\hline CYN & $-.22^{* *}$ & -.13 & -.12 & $-.25^{* *}$ & $-.29 * *$ & -.10 \\
\hline ASP & -.15 & -.01 & -.10 & $-.22^{* *}$ & -.30 ** & -.04 \\
\hline TPA & $-.26^{* *}$ & -.11 & -.13 & $-.31^{* *}$ & -.40 ** & -.10 \\
\hline LSE & -.50 ** & $-.57^{* *}$ & -.20 ** & $-.33^{* *}$ & $-.22^{* *}$ & $-.40 * *$ \\
\hline SOD & $-.33^{* *}$ & $-.43^{* *}$ & $-.21^{* *}$ & -.15 & .08 & $-.43^{* *}$ \\
\hline FAM & $-.23^{* *}$ & -.16 & -.13 & $-.19 * *$ & $-.20 * *$ & $-.23^{* *}$ \\
\hline WRK & $-.55^{* *}$ & $-.50 * *$ & $-.20 * *$ & $-.48 * *$ & -.36 ** & $-.45^{* *}$ \\
\hline TRT & $-.45^{* *}$ & $-.45^{* *}$ & $-.24^{* *}$ & $-.37^{* *}$ & $-.21 * *$ & $-.36^{* *}$ \\
\hline
\end{tabular}

${ }^{a} A N X$, Anxiety; FRS, Fears; OBS, Obsessiveness: DEP, Depression; HEA, Health Concerns; BIZ, Bizarre Mention; ANG, Anger; CYN, Cynicism; ASP, Antisocial Practices; TPA, Type A Behavior; LSE, Low Self-Esteem; SOD, Social Discomfort; FAM, Family Problems; WRK, Work Interference; and TRT, Negative Treatment Indicators. 'DINTRA, Intrapersonal; INTER, Interpersonal; ADAPT, Adaptation; STRES, Stress Management; MO0D, General Mood. ${ }^{* *} p<.01$.

MMPI-2 content scales for both the male and female nurses. For the male nurses, strong negative correlations, $<-.30$, are found to occur for Emotional Quotient Inventory total with the MMPI-2
Content scales of Depression, Type A Behavior, Low Self-Esteem, Work Interference, and Negative Treatment indicators. For the female nurses, even stronger correlations are observed for the Emotional 
Quotient Inventory total with the same MMPI-2 content scales, $<-.44$. The nature of the correlations with the External scales of the MMPI-2 suggest that individuals with a markedly low scores for emotional intelligence may possess symptoms of a depressed mood, fatigue, and pessimism; be harddriving, fast-moving, and irritable; have a very poor self-concept and give up easily; report a wide variety of attitudes and behaviors that are likely to contribute to poor work performance; and have a negative attitude with regard to the solvability of their problems. In addition, those female nurses with particularly low scores for emotional intelligence may also show symptoms of anxiety and nervousness, difficulties making decisions, shyness, and social introversion, as indicated by strong negative correlations with the Anxiety, Obsessiveness, and Social Discomfort content scales of the MMPI-2.

For both the male and female nurses, their scores on the Emotional Quotient Inventory composite scales of Stress Management and General Mood generally tended to correlate negatively with their scores on most of the MMPI-2 Content scales. For the female nurses, their scores on the Emotional Quotient Inventory composite scale of Adaptation also showed a significant negative relation to their scores on most of the MMPI-2 Content scales. For both the male and female nurses, their scores on the Intrapersonal and Interpersonal composite scales of the Emotional Quotient Inventory related negatively to only some of the MMPI-2 Content scales. Intrapersonal composite scale related most negatively to Depression, Low Self-Esteem, Social Discomfort, and Work Interference for both men and women; Interpersonal composite scale related most negatively to the same Content scales for women only and to Antisocial Practices for men.

In sum, nurses with a higher level of emotional intelligence show less psychopathology, as indicated by their scores for both the Content and Clinical scales of the MMPI-2. This finding was even stronger for the female than for the male nurses.

\section{Emotional Intelligence, Absenteeism Due to Illness, and Job Change}

To test whether nurses with high emotional intelligence have less absences due to illness and are better able to stay on the job, we computed the correlations between their Emotional Quotient Inventory total score, Emotional Quotient Inventory composite scale scores, the number of episodes of absence during a period of 2 years, the total number of days absent due to illness during a period of 2 years, the absence rate for the same period of 2 years, and job change. The results are summarized in Table 8. For both the male and female nurses, no significant correlations were observed for emotional intelligence with either absenteeism due to illness or job change. In other words, the amount of absenteeism due to illness and the probability of

Table 8 Emotional Quotient Inventory Scales: Correlation With Absenteeism by Gender

\begin{tabular}{lrrrrrr}
\hline & \multicolumn{5}{c}{ Emotional Quotient Inventory $^{\mathrm{a}}$} \\
\cline { 2 - 6 } Absenteeism & TOTAL & INTRA & INTER & ADAPT & STRES & M00D \\
\hline Men $(n=146)$ & & & & & & \\
$\quad$ Episodes of absence 2000 & .01 & .04 & .01 & .00 & .02 & -.06 \\
$\quad$ Absence rate 2000 & -.04 & .05 & -.18 & .01 & .00 &.- .01 \\
$\quad$ Episodes of absence 2001 & -.02 & .04 & .03 & -.02 & -.12 & -.05 \\
$\quad$ Absence rate 2001 & -.14 & -.18 & .02 & -.16 & -.05 & -.12 \\
Job change & -.09 & .03 & -.13 & -.07 & -.16 & -.08 \\
Women ( $n=234)$ & & & & & & \\
$\quad$ Episodes of absence 2000 & -.08 & -.06 & -.14 & -.05 & .04 & -.13 \\
Absence rate 2000 & -.08 & -.01 & -.10 & -.10 & -.13 & -.03 \\
$\quad$ Episodes of absence 2001 & .03 & .01 & .03 & .04 & .07 & -.01 \\
$\quad$ Absence rate 2001 & -.10 & -.06 & -.04 & -.06 & -.11 & -.13 \\
Job change & -.05 & -.02 & -.01 & -.02 & -.10 & -.06 \\
\hline
\end{tabular}

aINTRA, Intrapersonal; INTER, Interpersonal; ADAPT, Adaptation; STRES, Stress Management; MO0D, General Mood. 
job change within a period of 2 years was similar for nurses with a high versus low level of emotional intelligence.

\section{Discussion}

The results of the present study show that nurses working with extremely aggressive individuals with mental retardation have an above average level of emotional intelligence. A high Emotional Quotient Inventory total score indicates an individual who is in touch with his or her feelings, feels good about him/herself, and is fairly successful at realizing his or her potential. These same individuals tend to understand how others feel and are generally successful at relating to people. They are realistic, assertive, and fairly good at solving problems, good at stress management, and rarely lose control. The nurses in this sample also showed above average scores on the Emotional Quotient Inventory composite scales of Adaptability, Stress Management, and General Mood. The exact reason for the particularly high scores within this sample is unclear. It is possible that the homogeneity of our research group, which consisted of only nurses working with individuals who have mental retardation and severe behavior problems for at least 18 hours a week, may account for the elevated levels of emotional intelligence. This particular domain of work may simply attract emotionally intelligent people.

Some interesting gender differences were found. Although the male and female nurses showed roughly the same levels of emotional intelligence in general, remarkable differences were noted in their composite scale profiles. The male nurses scored significantly higher on the Intrapersonal composite scale and the Assertiveness and Self-Regard subscales. They also scored significantly higher on the Stress Management composite scale and the Stress Tolerance subscale. In other words, the male nurses in this sample appeared to be better able to express their feelings, thoughts, and beliefs and defend their rights than did the female nurses. The male nurses also tended to accept and respect themselves more, had a better sense of self-esteem, and felt more positively about themselves. Their tolerance of stress was also relatively higher, which means that they may be better able to withstand adverse events and stressful situations than would the female nurses. The male nurses were also calmer and rarely got overly anxious or agitated.
The female nurses, in contrast, scored significantly higher than did the male nurses on the Interpersonal composite scale and all of the associated subscales: Empathy, Interpersonal Relationship, and Social Responsibility. This suggests that they were more aware of the feelings of others and valued the feelings of others more than their male colleagues did. The female nurses tended to be better at the establishment and maintenance of mutually satisfying relationships, had a greater capacity for intimacy, and a better ability to give and receive affection. They also tended be more cooperative, contributing, and constructive members of the social group than did the male nurses in this sample.

The male and female nurses produced similar above-average scores on the Emotional Quotient Inventory Adaptability and General Mood composite scales, suggesting that they were quite flexible, realistic, and effective in dealing with problematic situations and also competent at achieving adequate solutions. In addition, they were basically able to enjoy their lives and experience an overall feeling of contentment. The significant but small differences in the domains of Interpersonal Relationships, Social Responsibility, and Tolerance of Stress for the male and female nurses studied here have also been detected in almost every other Emotional Quotient Inventory population sample examined throughout the world, and these differences may possibly explain why greater psychopathology is observed among men and greater anxiety-related disturbances among women (Bar-On, 1997).

In the present study, no gender-specific norms were applied. The nurses were compared to a general norm group. Nevertheless, the differences between the male and female nurses, particularly on the Emotional Quotient Inventory composite scales, can help us understand the gender-specific differences found to occur in the relations between emotional intelligence, coping, general intelligence, burnout, and psychopathology. If gender-specific norms had been used, however, these differences would not have stood out. For individual protocols, of course, both gender- and age-specific norms should be applied.

With respect to coping style, very similar results were obtained for the male and female nurses. In general, significant negative relations were found between emotional intelligence and the use of the depressive reaction and avoidance styles of coping. Conversely, a significant positive relation between emotional intelligence and the use of an active 
dealing style of coping was generally found. The female nurses in the sample had a positive relation between emotional intelligence and use of a social support seeking style of coping. This was not the case for the male nurses, which may be due to the differences in the interpersonal skills of male versus female nurses. In general, the above findings are consistent with previous research showing that more emotionally intelligent people adopt more active, problem-focused, and nonavoidant styles of coping. These same individuals tend to seek social support when they need it, and they are, therefore, less tempted to develop a depressive reaction when confronted with problems (Matthews \& Zeidner, 2000).

A small, but significant relation was found between emotional intelligence and general intelligence for the female but not the male nurses. This finding is consistent with the results of previous research by Davies, Stankov, and Roberts (1998) and Derksen, Kramer, and Katzko (2002), who found no meaningful relations between emotional intelligence and general intelligence. In other words, emotional intelligence clearly involves different abilities than does cognitive intelligence.

With respect to the relations between emotional intelligence and burnout, remarkable differences between the male and female nurses were encountered. Male nurses with lower scores for emotional intelligence, interpersonal skills, and general mood reported only fewer feelings of personal competence. They did not report greater feelings of emotional exhaustion or depersonalization. Female nurses with lower scores for emotional intelligence and all of the composite scales reported more symptoms of emotional exhaustion, depersonalization, and diminished personal accomplishment. The relation between lower emotional intelligence and the occurrence of symptoms of burnout was, thus, more obvious for female than for male nurses. This difference may explain the dissimilarities in the distributions of the burnout scales for the males versus females because significant differences in the average Emotional Quotient Inventory Intrapersonal, Interpersonal, and Stress Management composite scale scores for the male versus female nurses were also found.

With respect to the relations between emotional intelligence and the various aspects of psychopathology, the following can be concluded. Nurses with a higher level of emotional intelligence reported less psychopathology in terms of both the
MMPI-2 Content and Clinical scales. This relation was stronger for the female than the male nurses. A significant negative relation between the emotional intelligence scores and the MMPI-2 Scale 0 scores (Social Introversion) for both the male and female nurses was found, which shows that those nurses with a higher level of emotional intelligence, a better ability to express their feelings, better social skills, and a generally better mood score lower on the MMPI-2 scale, showing them to be more extraverted. Negative relations between emotional intelligence and the MMPI-2 Scales 2 and 7 (Depression and Psychoasthenia) were also found for the female nurses in particular. More specifically, lower scores for emotional intelligence in general, the Intrapersonal composite scale, and the General Mood composite scale were accompanied by higher scores on the MMPI-2 Scales 2 and 7, reflecting people who have negative feelings about themselves, tend to worry, feel depressed, and feel anxious at times. These same individuals tend to anticipate problems before they occur and to overreact to minor stress (Graham, 2000). For the male nurses, similar relations were not detected, which may stem from the relatively higher scores they attained on the Intrapersonal and Stress Management composite scales when compared to the female nurses.

Emotional intelligence was found to negatively relate to several of the MMPI-2 Content scales. Both the male and female nurses with particularly low emotional intelligence scores were found to show symptoms of depressed mood and fatigue, be hard-driving and irritable, have low self-confidence, display several symptoms of poor job employment, and believe that their problems are hard to solve. The female nurses with a particularly low level of emotional intelligence were also found to be particularly nervous and anxious, have difficulties making decisions, and be shy and socially introverted (Graham, 2000).

Finally, no significant relations were found between emotional intelligence and absence due to illness or job change for either of the sexes. Apparently, emotional intelligence does not play a role in absenteeism or the need to change jobs.

A general relation between emotional intelligence and adaptive success was found for both of the sexes but was more apparent for the female nurses. Possibly particular gender-specific skills, as seen in higher levels of Intrapersonal and Stress Management scores for the male nurses seem to be play a role in adaptive success. The results of this 
study, however, should be interpreted with caution. The cross-sectional design and correlational statistics do not allow us to draw sound conclusions regarding the direction of the causality for many of the observed relations and further longitudinal research is needed.

The strengths of the present study lie in the use of well-validated and thoroughly investigated estimates of emotional intelligence, coping, burnout, psychopathology, and general intelligence. In addition, the large sample size and high response rate reinforce the robustness of the findings.

The results of the present study have a number of implications for both future research and practical application. They provide further evidence for the construct of emotional intelligence and the utility of the Emotional Quotient Inventory for assessment purposes. The gender-specific nature of the results with regard to emotional intelligence make clear that future studies of nurses should consider males and females separately.

It may also be interesting in the future to address the prevention of job-related problems, burnout, or psychopathology on the basis of emotional intelligence profiles. Future researchers should also attempt to further clarify the gender-specific relations observed in the present study because these relations may play an important role in the emergence and, thus, prevention of both burnout and psychopathology. An important issue, which we did not investigate here, is the stability of emotional intelligence over time and the stability of the Emotional Quotient Inventory scores in particular. Longitudinal research is clearly needed to better ground the construct of emotional intelligence for contemporary assessment purposes. Finally, the present study concerned only nurses working a minimum of 18 hours a week; nurses with smaller contracts were not included, which means that further research is needed to validate the present results with a more varied subject sample.

In closing, these findings have two major implications for actual practice. First, the relevance of the Emotional Quotient Inventory as an instrument for training a variety of professionals should be clear. The results of the present research, together with those of the larger research program this study is part of, are a basis for individual training programs for those nurses working with clients who have mental retardation and severe behavior problems. The results of the Emotional Quotient Inventory provide clearer insight into what aspects of per- sonality contribute to successful functioning and what aspects relate to nonadaptability. Because Emotional Quotient Inventory aspects are trainable skills, nurses can be adequately coached on the specific skills they need for their professional work environment. Second, the focus of the training for professionals and certainly nurses working under stressful conditions should probably be different for males and females. Female nurses may need more extensive training in the areas of intrapersonal skills and stress management than do male nurses, for example, in order to avoid the otherwise resultant burnout. In addition to the benefits for the health care personnel themselves, their coworkers, and their employers, prevention of burnout will also have positive effects for the clients with severe behavior problems in question. Qualified and stable teams of caregivers are necessary preconditions for treatment intervention programs or ways to improve safe environments for all people involved.

\section{References}

Averill, J. R. (2000). Intelligence, emotion and creativity: From trichotomy to trinity. In R. BarOn \& J. D. A. Parker (Eds.), The handbook of emotional intelligence: Theory, development, assessment, and application at home, school, and in the workplace (pp. 277-298). San Francisco: Jossey-Bass.

Bar-On, R. (1997). Bar-On Emotional Quotient Inventory: Technical manual. Toronto: Multi Health Systems.

Bar-On, R., Brown, J. M., Kirkcaldy, B. D., \& Thomé, E. P. (2000). Emotional expression and implications for occupational stress; an application of the Emotional Quotient Inventory (EQI). Personality and Individual Differences, 28, 1107-1118.

Bromley, J., \& Emerson, E. (1995). Beliefs and emotional reactions of care staff working with people with challenging behaviour. Journal of Intellectual Disability Research, 39, 341-352.

Butcher, J. N., Dahlstrom, W. G., Graham, J. R., Tellegen, A., \& Kaemmer, B. (1989). Minnesota Multiphasic Personality Inventory (MMPI-2): Manual for administration and scoring. Minneapolis: University of Minnesota Press.

Davies, M., Stankov, L., \& Roberts, R. D. (1998). Emotional intelligence: In search of an elusive construct. Journal of Personality and Social Psychology, 75, 989-1015. 
Dawda, D., \& Hart, S. D. (2000). Assessing emotional intelligence: Reliability and validity of the Bar-On Emotional Quotient Inventory (EQI) in university students. Personality and Individual Differences, 28, 797-812.

Derksen, J. J. L., Egger, J., \& Bögels, T. J. P. M. (1998). GAMA opgavenboek [GAMA test book]. Nijmegen, The Netherlands: PEN Tests.

Derksen, J. J. L., Jeuken, J., \& Klein Herenbrink, A. J. M. (1998). Bar-On Emotioneel Quotiënt Vragenlijst, Nederlandse vertaling en bewerking [Bar-On Emotional Quotient Inventory, Dutch translation and adaptation]. Nijmegen, The Netherlands: PEN Tests.

Derksen, J. J. L., Kramer, I. M. A., \& Katzko, M. (2002). Does a self report measure for emotional intelligence assess something different than general intelligence? Personality and Individual Differences, 32, 37-48.

Derksen, J. J. L., Mey, H. R. A. D., Sloore, H., \& Hellenbosch, G. (1995). MMPI-2: Handleiding bij afname, scoring en interpretatie (2nd ed.) [MMPI-2: Manual for administration, scoring, and interpretation]. Nijmegen, The Netherlands: PEN Tests.

Didden, R., Duker, P. C., \& Korzilius, H. (1997). Meta-analytic study on treatment effectiveness for problem behaviors with individuals who have mental retardation. American Journal on Mental Retardation, 101, 387-399.

Goleman, D. (1995). Emotional intelligence. New York: Bantam Books.

Graham, J. R. (2000). MMPI-2. Assessing personality and psychopathology. New York: Oxford University Press.

Kramer, G. J. A. (1995). Consensus Protocol Ernstig Probleemgedrag [Consensus Protocol Severe Problem Behavior]. Utrecht, The Netherlands: Nationaal Ziekenhuis Instituut.

Lassiter, K. S., \& Matthews, T. D. (1999). Testretest reliability of the General Ability Measure for Adults. Perceptual and Motor Skills, 88, 531534.

Lazarus, R. S., \& Folkman, S. (1984). Stress, appraisal and coping. New York: Springer.

Leiter, M. P., \& Maslach, C. (1988). The impact of interpersonal environment on burnout and organizational commitment. Journal of Organizational Behavior, 9, 297-308.

Maslach, C., \& Jackson, S. E. (1986). Maslach
Burnout Inventory manual (2nd ed.). Palo Alto, CA: Consulting Psychologists Press.

Matthews, G., \& Zeidner, M. (2000). Emotional intelligence, adaptation to stressful encounters, and health outcomes. In R. Bar-On \& J. D. A. Parker (Eds.), The handbook of emotional intelligence: Theory, development, assessment, and application at home, school, and in the workplace (pp. 459-489). San Francisco: Jossey-Bass.

Mayer, J. D., \& Salovey, P. (1993). The intelligence of emotional intelligence. Intelligence, 17, 433442.

Naglieri, J. A., \& Bardos, A. N. (1997). General Ability Measure for Adults (GAMA). Minneapolis: NSC.

Salovey, P., Hsee, C. K., \& Mayer, J. D. (1993). Emotional intelligence and the self-regulation of affect. In D. M. Wegner \& J. W. Pennebaker (Eds.), Handbook of mental control (pp. 258277). Englewood Cliffs, NJ: Prentice Hall.

Salovey, P., \& Mayer, J. D. (1990). Emotional intelligence. Imagination, Cognition and Personality, 9, 185-211.

Schaufeli, W. B., \& Dierendonck, D. V. (2000). Handleiding Utrechtse Burnout Schaal [Manual Utrecht-Burnout Scale]. Lisse, The Netherlands: Swets Test Publishers.

Schreurs, P. J. G., Willige, G. V. D., Brosschot, J. F., Tellegen, B., \& Graus, G. M. H. (1993). Utrechtse coping lijst: omgaan met problemen en gebeurtenissen, herziene handleiding [UtrechtCoping List: Coping with problems and events-Revised manual]. Lisse, The Netherlands: Swets \& Zeitlinger.

Received 10/09/02, first decision 3/24/03, accepted 4/28/03

Editor-in-charge: Steven J. Taylor

This study was supported in part by grants from ZONMw and PEN Tests Publisher.

\footnotetext{
Authors:

Linda Gerits, PhD (E-mail: L.Gerits@psych.kun. nl), Senior Researcher; Jan J. L. Derksen, PhD (Email: J.Derksen@psych.kun.nl), Professor; and Antoine B. Verbruggen, MA, Research Assistant, Department of Clinical Psychology, University of Nijmegen, PO Box 9104, 6500 HE Nijmegen, The Netherlands.
} 